\title{
Green Coffee Bean Extract as a Weight Loss Supplement
}

\author{
Mehnoosh Samadi ${ }^{1}$, Majid Mohammadshahi ${ }^{2}$ and Fatemeh Haidari ${ }^{\star}$
}

${ }^{1}$ Department of Nutrition, Paramedical School, Ahvaz Jundishapur University of Medical Sciences, Ahvaz, Iran

${ }^{2}$ Nutrition and Metabolic Diseases Research Centre, Ahvaz Jundishapur University of Medical Sciences, Ahvaz 61357-15794, Iran

\begin{abstract}
Background: Weight management is a long-standing goal of achieving a healthy lifestyle. Therefore, it is important to find a safe and effective way to reduce the body weight of overweight or obese people. In this regard, studies focusing on the components of the diet are very important.

Methods: We reviewed the results of the studies assessing the efficacy of green coffee extract (GCE) as a weight-reducing agent.

Results: The components of the diet may work synergistically to prevent or promote weight management.

Conclusions: Recently, green coffee introduced as the richest sources of cholorogenic acid that can play a role in weight loss.
\end{abstract}

Keywords: Obesity; Weight management; Green coffee; Cholorogenic acid

\section{Introduction}

Obesity has been increasing rapidly in recent years and now has become a public health problem in the word [1]. Obesity is defined as excess accumulation of body fat and is known as a low grade inflammation condition [2]. Obesity, not only affect the body image and self-esteem but also it is associated with increased risk for numerous chronic diseases, including hypertension, insulin resistance, type 2 diabetes and cardiovascular disease [3,4]. Therefore, it is important to find a safe and effective way to manage body weight especially in obese patients. Weight management is a long-standing goal of achieving a healthy life that is including healthy eating and physical activity to maintain a balance between intake and energy consumption [1]. However, several strategies have been used to treat obesity and its related complications. Behavioural therapy, surgery and drug therapy are the most usual treatment of obesity [5]. Since human diets contain many different components that may act synergistically to prevent or promote overweight and obesity, studying the components of the diet may be an informative strategy to assess their efficacy and underlying mechanisms [3].

Recently, issues of high costs and potential adverse effects of most weight loss drugs have led obese patients to try nutraceuticals that may cause weight loss [6,7]. Coffee is one of the most commonly consumed beverages in the world that its beneficial effects on human health have been a subject of many studies [8-12]. One of the common traditional forms of coffee is green coffee extract (GCE) that prepared from green or raw (unroasted) coffee been. It is also present in roasted coffee, but much of the GCE is destroyed during the roasting process [13]. GCE has been introduced as the richest sources of cholorogenic acid [13-16] and most of weight losing effects of GCE has proposed to be related to its cholorogenic acid content [17]. Cholorogenic acid (CGA) is a natural chemical compound which is the ester of caffeic acid and quinic acid. It is an important biosynthetic intermediate [13]. Cholorogenic acid is an important intermediate in lignin biosynthesis. This compound, known as an antioxidant, may also slow the release of glucose into the bloodstream after a meal [17]. Isomers of cholorogenic acid are found in potatoes [18] and cholorogenic acid is the most abundant phenolic acid in the flesh of eggplant fruits [19]. It is also found in prunes [20] but the richest source of cholorogenic acid is green coffee bean extract
[13]. Therefore, the objective of this paper was to review the results of the studies assessing the efficacy of GCE as a weight loss supplement.

\section{Methods}

In this review study, the results of the studies assessing the efficacy of green coffee extract (GCE) as a weight-reducing agent were reviewed. This review study was done by searching on PubMed, Scopus and Science Direct by entering "Obesity", "Weight management", "Green Coffee, "Cholorogenic acid" as keywords. In this study 14 inclusive clinical trial articles and review articles which were published during 2006 to 2014 were reviewed. Among these articles, 7 articles were in humans and 7 articles were in animals.

\section{Results}

The efficacy of green coffee extract in animal and human studies. In some studies effect on low calorie diet supplemented with nutraceuticals component reported that can be effective in weight management [21]. In animal studies that conducted to investigate the effects of green coffee extract on body weight, inconsistent results were reported. Cheong LK showed that high fat diet (HFD) supplemented with $0.5 \% \mathrm{w} / \mathrm{w}$ GCE did not attenuate weight gain in obese mice after 12 weeks [22]. However the results of the Shimoda study suggested that oral administration of $0.5 \%$ and $1 \%$ GCE was effective against weight gain and visceral fat accumulation in mice were fed with a standard diet for 14 days [23].

In another study conducted by Song, these investigators showed that decaffeinated green coffee bean extract reversed HFD-induced fat accumulation and insulin resistance in a dose dependent manner.

*Corresponding author: Fatemeh Haidari, Nutrition and Metabolic Diseases Research Centre, Ahvaz Jundishapur University of Medical Sciences, Ahvaz 61357-15794, Iran, Tel: +98 613 3339092, Fax: +98 613 3738330; E-mail: haidari58@gmail.com

Received November 18, 2015; Accepted December 12, 2015; Published December 17, 2015

Citation: Samadi M, Mohammadshahi M, Haidari F (2015) Green Coffee Bean Extract as a Weight Loss Supplement. J Nutr Disorders Ther 5: 180. doi:10.4172/2161- 0509.1000180

Copyright: $\odot 2015$ Samadi M, et al. This is an open-access article distributed unde the terms of the Creative Commons Attribution License, which permits unrestricted use, distribution, and reproduction in any medium, provided the original author and source are credited. 
This study supposed that the positive effect of decaffeinated GCE may be due to the down regulating of genes involved in adipogenesis and inflammation in visceral adipose tissue [17].

Several human clinical trials have also conducted to examine the efficacy and safety of commercial GCE at reducing weight and body mass in overweight and obese adults [24-28]. The results from a clinical study performed on 30 overweight people showed consumption of instant coffee enriched with cholorogenic acid for 12 -week induced a significant reduction in body mass index and body fat, compared with the control [26]. In 2012, Vinson in a randomized, double-blind, placebo-controlled, crossover study administrated high-dose GCA (1050 mg), low-dose GCA (700 mg), or placebo to 16 overweight adults in separate six-week treatment periods followed by two-week washout periods. The results of the study showed significant reductions in body weight, body mass index, and percent body fat, but with no significant alterations in dietary intakes over the course of the study. Finally, this study suggested that commercial green coffee extract product GCA" might be an effective nutraceuticals in reducing weight in pre-obese adults, and might be an inexpensive means of preventing obesity in overweight adults [28]. The results of a systematic review and metaanalysis of randomized clinical trials revealed a significant difference in body weight in GCE compared with placebo (mean difference: $-2.47 \mathrm{~kg} ; 95 \%$ CI: $-4.23,-0.72$ ). This review study concluded that the results of these trials are promising, but the studies are all of poor methodological quality [24]. Recently, Cornelis has also emphasized that the inter individual variation in the physiological exposure or response to any of the many chemicals present in coffee may affect the persistence and magnitude of its health effects. This study concluded that only subgroups of overweight patients most likely to benefit from increasing green coffee consumption [25].

\section{Discussion}

\section{How does green coffee extract work for weight loss?}

Studies have shown that green coffee enhances energy metabolism and reduces lipogenesis by down regulating SREBP-1c (Sterol regulatory element-binding protein) and related molecules, which leads to the suppression of body fat accumulation [11]. Scientific theories expressed that natural substances found in green coffee prevent weight gain and fat accumulation by inhibiting fat absorption and increasing fat metabolism in liver $[23,29]$. Supplementation with coffee polyphenols can also be effective in weight loss and reduce abdominal and liver fat accumulation by inhibition of macrophages infiltration into adipose tissues [11].

The inhibition of glucose-6-phosphatase (Glc-6-P) hydrolysis in intact human liver microsomes by decaffeinated green coffee extract was also shown in vitro study. Glc-6-P exists primarily in the liver and catalyses the end step in gluconeogenesis and glycogenolysis. The inhibition of this enzyme by green coffee may contribute to its antidiabetic and weight loss effects by reducing hepatic glucose production [30].

It seems that the beneficial effects of green coffee in relation to weight loss attributed to its cholorogenic acid content $[28,29]$. Cholorogenic acids have high bioavailability in humans [30,31]. Because of the synergistic effect between caffeine and cholorogenic acid in green coffee extract, green coffee extract was shown to suppress body weight gain better than cholorogenic acid or caffeine alone $[32,33]$.

As mentioned above, animal studies on the effectiveness of green coffee as a weight loss supplement have provided conflicting results.
In human studies, the effect of green coffee has also been examined without controlling of diet and physical activity. Moreover, none of the studies have examined the effect of green coffee on body composition changes, muscle mass maintenance, serum adipocytokins levels and obesity- related metabolic parameters. Therefore, future investigations with assessing of lifestyle changes such as physical activity levels and dietary intake are required in this field.

\section{Conclusion}

Therefore, more rigorous trials are needed to assess the usefulness of green coffee extract as a weight loss tool. Especially, According to the effect of green coffee on increasing energy metabolism and reducing lipogenesis, it is recommended that studies be conducted on patients with BMI more than 40. Moreover, it is imperative that future studies of green coffee and health account for individual variations in the physiological response to natural components present in green coffee.

\section{References}

1. Dunn D (2015) Addressing the Problem of Obesity. AORN J 102: 111-115

2. Mohammadshahi M, Haidari F, Karandish M, Ebrahimi S, Haghighizadeh MH (2014) A Randomized Clinical Trial of Nutrition Education for improvement of Diet Quality and Inflammation in Iranian Obese Women. J Nutr Metab 605782.

3. Haidari F, Shirbeigi E, MakanCheraghpour, Mohammadshahi M (2014) Association of dietary patterns with body mass index, waist circumference, and blood pressure in an adult population in Ahvaz, Iran. Saudi Med J 35: 967-974

4. Finucane MM, Stevens GA, Cowan MJ, Danaei G, Lin JK et al. (2011) National, regional, and global trends in body-mass index since 1980: systematic analysis of health examination surveys and epidemiological studies with 960 countryyears and 9.1 million participants. Lancet 377: 557-567.

5. Salem Z, Rezaeian M (2011) Interventional drug therapy. Zahedan J Res Med Sci 10: 41-57.

6. Ormsbee MJ, Rawal SR, Baur DA, Kinsey AW, Elam ML, et al. (2014) The effects of a multi-ingredient dietarysupplement on body composition adipokines,blood lipids, and metabolic health in overweight and obese men and women: a randomized controlled trial. J Int Soc Sports Nutr 11: 37-47.

7. Tverdal A, Skurtveit S (2003) Coffee intake and mortality from liver cirrhosis. Ann Epidemiol 13: 419-423.

8. Van Dam RM, Hu FB (2005) Coffee consumption and risk of type 2 diabetes: a systematic review. JAMA 294: 97-104

9. Greenberg JA, Boozer CN, Geliebter A (2006) Coffee, diabetes, and weight control. Am J Clin Nutr 84: 682-693.

10. Tanaka K, Shoko N, Shizuka T, Mihoko K, Hiroshi S, et al. (2009) Anti-obesity and hypotriglyceridemic properties of coffee bean extract in SD rats. Food Sci Technol Res 15: 147-152.

11. MuraseT, Misawa K, Minegishi $Y$, Aoki M, Ominami H, et al. (2011) Coffee polyphenols suppress diet-induced body fat accumulation by downregulating SREBP-1c and related molecules in C57BL/6J mice. Am J Physiol Endocrino Metab 300: 122-133.

12. Ho L, Varghese M, Wang J, Zhao W, Chen F, et al. (2012) Dietary supplementation with decaffeinated green coffee improves diet-induced insulin resistance and brain energy metabolism in mice. Nutr Neuroscience 15: 37-45

13. Higdon JV, Frei B (2006) Coffee and health: a review of recent human research. Crit Rev Food Sci Nutr 46: 101-123.

14. Inoue M, Kurahashi N, Iwasaki M, Shimazu T, Tanaka Y (2009) Effect of coffee and green tea consumption on the risk of liver cancer: cohort analysis by hepatitis virus infection status. Cancer Epidemiol Biomarkers Prev 18: 17461753.

15. Andrade KS, Goncalvez RT, Maraschin M, Ribeiro-do-Valle RM, Martinez J, et al. (2012) Supercritical fluid extraction from spent coffee grounds and coffee husks: antioxidant activity and effect of operational variables on extract composition. Talanta 88: 544-552.

16. Watanabe T, Arai Y, Mitsui Y, Kusaura T, Okawa W, et al. (2006) The blood 
Citation: Samadi M, Mohammadshahi M, Haidari F (2015) Green Coffee Bean Extract as a Weight Loss Supplement. J Nutr Disorders Ther 5: 180. doi:10.4172/2161-0509.1000180

pressure-lowering effect and safety of chlorogenic acid from green coffee bean extract in essential hypertension. Clin Exp Hypertens 28: 439-449.

17. Song SJ, Choi S, Park T (2014) Decaffeinated Green Coffee Bean Extract Attenuates Diet-Induced Obesity and Insulin Resistance in Mice. Evid Based Complement Alternat Med 2014: 718379.

18. Friedman M (1997) Chemistry, Biochemistry, and Dietary Role of Potato Polyphenols. A Review. J Agric Food Chem 45: 1523-1540.

19. Devanand L, Mukhopadhyay L, Mukhopadhyay S (2006) Influence of Sample Preparation on Assay of Phenolic Acids from Eggplant. J Agric Food Chem 54: 41-47.

20. Stacewicz-Sapuntzakis M, Bowen PE, Hussain EA, Damayanti-Wood BI, Farnsworth NR (2001) Chemical composition and potential health effects of prunes: a functional food? Crit Rev Food Sci Nutr 41: 251-286.

21. Mohammadshahi M, Rafiei H, KarandishM, Omidian K, Haidari F (2012) Effect on calorie restriction supplemented with Genistein on serum levels of glucose lipid profile and inflammatory markers (Resistin and hsCRP) in obese rats. Asian J Biochem 7: 98-105.

22. Cheong JD, Croft KD, Henry PD, Matthews V, Hodgson JM, et al. (2014) Green coffee polyphenols do not attenuate features of the metabolic syndrome and improve endothelial function in mice fed a high fat diet. Arch Biochem Biophys 559: $46-52$.

23. Shimoda H, Seki E, Aitani M (2006) Inhibitory effect of green coffee bean extract on fat accumulation and body weight gain in mice. BMC Complement Altern Med 6: 9-15.

24. Onakpoya I, Terry R, Ernst E (2011) The use of green coffee extract as a weight loss supplement: a systematic review and meta-analysis of randomised clinical trials. Gastroenterol Res Pract 2011: 382852.

25. Cornelis MC (2015) Toward systems epidemiology of coffee and health. Curr Opin Lipidol 26: 20-29.
26. Thom E (2007) The effect of chlorogenic acid enriched coffee on glucose absorption in healthy volunteers and its effect on body mass when used longterm in overweight and obese people. J Int Med Res 35: 900-908.

27. Revuelta-Iniesta R, Al-Dujaili EA (2014) Consumption of green coffee reduces blood pressure and body composition by influencing $11 \beta$-HSD1 enzyme activity in healthy individuals: a pilot crossover study using green and black coffee. Biomed Res Int 2014: 482704.

28. Vinson J, Burnham B, Nagendran MV (2012) Randomized, double-blind, placebo-controlled, linear dose, crossover study to evaluate the efficacy and safety of a green coffee bean extract in overweight subjects. Diabetes Metab Syndr Obes 5: 21-27.

29. Meng S, Cao J, Feng Q, Peng J, Hu Y (2013) Roles of Chlorogenic Acid on Regulating Glucose and Lipids Metabolism: A Review. Evid Based Complement Alternat Med 2013: 801457.

30. Henry-Vitrac C, Ibarra A, Roller M, Merillon JM, Vitrac X (2010) Contribution of chlorogenic acids to the inhibition of human hepatic glucose-6-phosphatase activity in vitro by Svetol, a standardized decaffeinated green coffee extract. J Agric Food Chem 58: 4141-4144.

31. Farah A, Monteiro M, Donangelo CM, Lafay S (2008) Cholorogenic Acids from Green Coffee Extract are Highly Bioavailable in Humans. J Nutr 138: 2309 2315.

32. Suzuki A, Fujii A, Jokura H, Tokimitsu I, Hase T, et al. (2008) Hydroxyhydroquinone interferes with the chlorogenic acid-induced restoration of endothelial function in spontaneously hypertensive rats. Am J Hypertens 21: 23-27.

33. Zheng G, Qiu Y, Zhang QF, Li D (2014) Cholorogenic acid and caffeine in combination inhibit fat accumulation by regulating hepatic lipid metabolismrelated enzymes in mice. Br J Nutr 112: 1034-1040. 